\title{
Start up of the EGSB reactor under the condition of poor nutrition and Microbial community analysis by next generation sequencing
}

\author{
Liao Runhua', a, Jiang Yan ${ }^{1, b}$, Wang Weipan ${ }^{1, c}$, Du Jie ${ }^{1, d}$, Li Yueming ${ }^{1, \mathrm{e}}$ and Fan \\ $\operatorname{Bin}^{1, f}$ \\ ${ }^{1}$ Jingdezhen Ceramic Institute, Jingdezhen 333403, China \\ amaxlrh@126.com, ${ }^{b} 519663575 @ q q . c o m,{ }^{c} 1306617315 @ q q . c o m,{ }^{d}$ sallydj@126.com, \\ 'lym6329@126.com, $519663575 @ q q . c o m$
}

Keywords: Start up; EGSB reactor; Microbial community; next generation sequencing

Abstract: The objective of this study was to investigate the successful start-up of poor nutrition simulated wastewater with sodium acetate carbon source in an EGSB reactor, and EGSB reactor inoculated the flocculent sludge from the anoxic zone in the municipal sewage treatment plant. The results demonstrated the COD removal efficiency can also achieve more than $80 \%$, even reaching above $95 \%$, when the COD concentration of influent was improved from 200, 500 to $1000 \mathrm{mg} / \mathrm{L}$ in the continuous operation of 73 days. Furthermore, the microbial communities of seeding source, sludge samples collected on the 43th day (A1) and at the end of operation (A2) were investigated with the next generation sequences (NGS) tool in the EGSB reactor. Analysis results showed that the microbial diversity decreased. At the bacterial phylum level, there was an apparent increase in relative sequences of Chloroflexi and Euryarchaeota (33.01\% and $0.63 \%$ in A1, and $44.03 \%$ and $7.14 \%$ in A2), which was $19.40 \%$ and $0.54 \%$ in the inoculum. At the genus level, except unclassified genus, Methanosarcina, Clostridium and WCHB1-05 were found to be the main members, whose sum occupied $16.13 \%$ and $18.41 \%$ of the total sequences in A1 and A2, respectively.

\section{Introduction}

The expanded granular sludge bed (EGSB) reactor is the third generation anaerobic bioreactor developed on the basis of the Up-flow Anaerobic Sludge Blanket (UASB) reactor ${ }^{[1]}$. Compared with UASB reactor, it increases the part of influent recycling, which causes that the rising velocity of the liquid in the reactor is much higher than that of UASB reactor, and that sewage and microbial strengthen the contact between them. Furthermore, it is because of the unique technical advantages that the EGSB reactor can be used for a variety of organic wastewater treatment, and has high processing efficiency ${ }^{[2-4]}$. So far, in the domestic and foreign literature, EGSB reactor sludge were mostly adopt cultivating granular sludge in UASB reactor, and mainly treated the rich nutrition high concentration wastewater. However, it was rarely reported that poor nutrition low concentration wastewater was treated in an EGSB reactor. In the present study, EGSB reactor was adopt the flocculent sludge from the anoxic zone in the municipal sewage treatment plant, and the start-up of poor nutrition simulated wastewater with sodium acetate carbon source in an EGSB reactor was investigated. At the same time, the microbial communities of seeding source, sludge samples collected on the 43th day (A1) and at the end of operation (A2) were investigated with the next generation sequences (NGS) tool in the EGSB reactor.

\section{Material and methods}

\section{Bioreactor, inoculation and synthetic wastewater}

The schematic diagram of the bench-scale EGSB reactor used in this study is shown in Fig. 1. The Plexiglas EGSB reactor was $60 \mathrm{~mm}$ in diameter and $1100 \mathrm{~cm}$ in height, giving a total volume of $3.58 \mathrm{~L}$ and a working volume of $2.31 \mathrm{~L}$. A peristaltic pump was used to introduce influent at the column bottom of the reactor. A gas-washing device was used to collect the generated $\mathrm{CH}_{4}$ and $\mathrm{CO}_{2}$ gas at the column top. A 
three-phase separator was installed at the reactor top to keep the biomass within the reactor. Excess sludge was discharged from the bottom of the EGSB reactor. The liquid up-flow velocity was controlled by inner recirculation. The EGSB reactor was operated under mesophilic conditions (35 \pm $\left.1^{\circ} \mathrm{C}\right){ }^{[5]}$ and its temperature was maintained by a water bath.

The seed sludge was domesticated from the anoxic sludge (mixed liquor volatile suspended solids (MLVSS):27.178 g/L) of the Fuliangxian Urban Sewage Treatment Plant, Jingdezhen, China, which was filtered through $0.2 \mathrm{~mm}$ Tyler mesh to eliminate most grit. The reactor was started up with the influent COD concentration at about $200 \mathrm{mg} / \mathrm{L}$, and liquid up-flow velocity at $2.5 \mathrm{~m} / \mathrm{hr}$, hydraulic

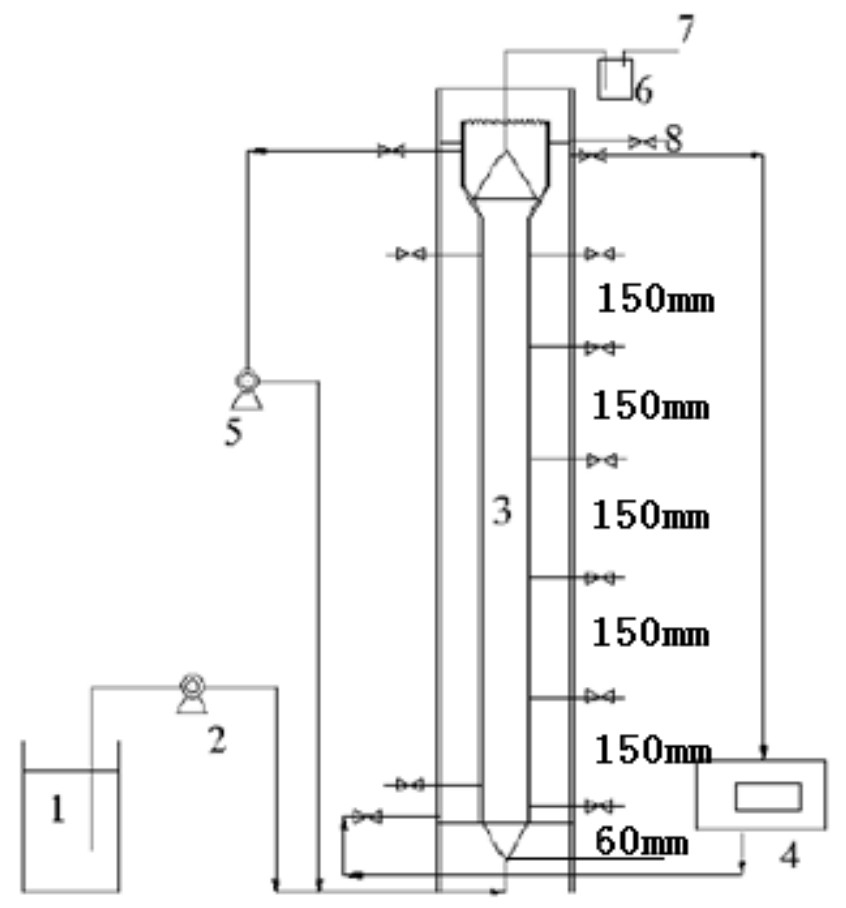
retention time (HRT) (22 h), and sodium acetate as carbon source. During 73 days of operation, the influent COD concentration of the synthetic wastewater gradually increased from 200 to 500, and $1000 \mathrm{mg} / \mathrm{L}$. Simulated oligotrophic wastewater was made of sodium acetate as the substrate and tap water as solvent configuration, not to add other nutritional elements.

Fig. 1. Experimental apparatus and process flow chart of the EGSB reactor operated at $35 \pm 1^{\circ} \mathrm{C}$, in which: (1) Influent tank; (2) Influent pump; (3) EGSB reactor (4) Water bath temperature control system;(5) Recycling pump; (6) gas sampler; (7) wet gas meter; (8) effluent water;

\section{Chemical analysis}

Wastewater parameters chemical oxygen demand (COD), Mixed Liquor Suspended Solids (MLSS) and Mixed Liquor Volatile Suspended Solids (MLVSS) were measured according to the Standard Method $^{[6]}$. A pH meter (pHS-25) was used to determine the $\mathrm{pH}$ values of liquid samples and Oxidation-Reduction Potential (ORP) were performed with the use of a MP551 conductivity meter (SANXIN Instrument and Meter Plant, Shanghai, China).

\section{Microbial community analysis}

The samples were subjected to total genomic DNA extraction using the Power Soil DNA extraction kit (Mo Bio, Carlsbad, CA) following the manufacture's procedure. Preparation of 16S rRNA gene amplicon and subsequent Illumina sequencing were conducted by the DNA Services Facility at the University of Illinoisat Chicago. Briefly, PCR amplification were conducted in triplicate reactions for all samples using the 515F/907R primer set that amplifies the V4-V5 region of the 16SrRNA gene ${ }^{[7-8]}$. Amplicons of the triplicate PCR reactions were pooled and then sequenced using an Illumina HiSeq 2000 instrument. De novo assemble of paired-end reads was performed with the software package CLC Genomics Workbench Version 6.0 (CLC bio, Cambridge, MA), and the resulting fastq files were trimmed using a cutoff quality score of Q15 and read length of larger than $200 \mathrm{bp}^{[9]}$. Only the forward reads were used for downstream analysis, as it was reported that including the reverse reads add little additional information $^{[10]}$.

After sequencing, the raw fastq files were demultiplexed and quality-filtered using QIIME (version 1.17). As the number of spurious phylotypes increases with sequencing effort, equal number of sequence 
reads should be used to compare microbial community among samples to minimize the sequencing $\operatorname{artifact}^{[11]}$. In this work,40000 reads from every sample were randomly picked and grouped into operational units (OTUs) with $97 \%$ similarity cutoff using UPARSE (version 7.1 http://drive5.com/uparse/). Chimeric sequences were identified and removed using UCHIME. Community diversity index (Chao1, PD, Simpson, Shannon diversity index) and rarefaction curves were generated using the MOTHUR program. The taxonomic assignment of OTUs was performed by RDP Classifier (http://rdp.cme.msu.edu/) against the silva (SSU115) 16S rRNA database at 70\% threshold $^{[12]}$.

\section{Results and discussion}

\section{Start-up performance}

The change of ORP, $\mathrm{pH}$ value and COD removal efficiency was shown in Figure 2 during the start-up process of the EGSB reactor. As can be seen from the figure 2, the COD removal efficiency reached more than $80 \%$ after the continuous operation of 15 days, which indicated that it was good adaptability for the poor nutrition simulated wastewater treatment in an EGSB reactor. In the meantime, the COD removal efficiency can also achieve more than $80 \%$, even reaching above $95 \%$, when the COD concentration of influent was improved from 200, 500 to $1000 \mathrm{mg} / \mathrm{L}$ in the continuous operation of 73 days. At this time, it was successful that the EGSB reactor started up. In the start-up process of the EGSB reactor, the $\mathrm{pH}$ value of influent, in the reactor and effluent ranged between 7.2 to 8.4, and the Oxidation Reduction Potential of effluent and in the reactor was between $0 \sim-250 \mathrm{mv}$. The change trend of COD, $\mathrm{pH}$ and ORP was similar results with the previously reported literature ${ }^{[13-14]}$.
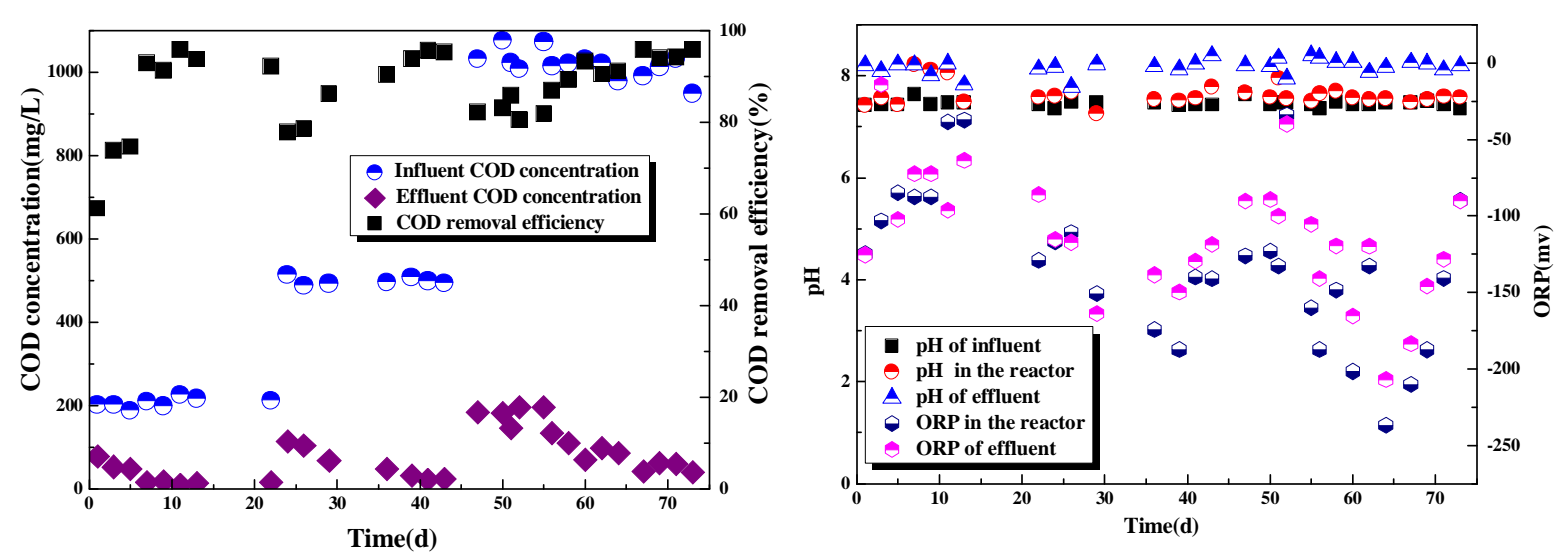

Fig. 2. Removal efficiency of COD, $\mathrm{pH}$ and ORP during the start-up and operation of an EGSB reactor

\section{Microbial community}

To reveal the shift in the microbial community structure during the start-up process of the EGSB reactor, the inoculum, and sludge samples collected on the 43th day (A1) and at the end of operation (A2) were used to pyrosequence the former region of the $16 \mathrm{~S}$ rRNA gene using a 454 GS-FLX sequencer. The sampling was prepared as a mixture of $10 \mathrm{~mL}$ mixed liquor at three ports $(60 \mathrm{~mm}, 210 \mathrm{~mm}$, and $360 \mathrm{~mm}$ from the bottom). A total of $22049716 \mathrm{~S}$ rRNA sequence reads were generated by the pyrosequencing of duplicate samples. After filtering the low quality reads using the RDP Initial Process in Pyrosequencing Pipeline (PP) and trimming the adapters, barcodes and primers, denoising, filtering out chimeras, the library size of the sample was normalized to 133114 high quality sequence reads and 4581 operational taxonomic units(OTUs) with similarity cutoffs of $3 \%$ were obtained from a single lane of an eight-lane pico-titer plate on the Genome Sequencer FLX Titanium system.

The sequencing depths were adequate to capture majority of the microbial diversity, as indicated by the rare faction curves approaching the asymptotic state. Microbial communities at the different stages of the 
anaerobic digestion processes all showed very diverse microbial communities (Table 1). The Shannon index (H') for three samples ranged from 7.8 to 8.4, and showed a downward trend. And that variational trend of Simpson diversity index was the same as Shannon index from the Table 1. While variational trend of OTUs, Chao1, and PD increased first and then decreased, it indicated that the microbial diversity decreased with the increase of the influent load, after the stable operation of the EGSB reactor.

Table.1. Raw, effective bacterial reads, and plus numbers of OTUs, Chao1, PD, Shannon and Simpson of three activated sludge samples at level of 3\% cutoff. (A1 = samples were taken after 43rd days of an

EGSB operation, and A2 = samples were taken at the end of an EGSB operation).

\begin{tabular}{cccccccc}
\hline Sample & $\begin{array}{c}\text { Raw } \\
\text { reads }\end{array}$ & $\begin{array}{c}\text { Effective } \\
\text { reads }\end{array}$ & OTUs & chao1 & PD & shannon & simpson \\
\hline Inoculum & 72707 & 41635 & 1546 & 1770 & 108 & 8.4 & 0.99 \\
A1 & 72239 & 42933 & 1595 & 1832 & 111 & 8.3 & 0.99 \\
A2 & 75551 & 48546 & 1440 & 1763 & 103 & 7.8 & 0.98 \\
\hline
\end{tabular}

Note: OTUs: operational taxonomic units; Shannon: Shannon's diversity index;

Chao-community richness. A higher number represents more richness;

Shannon - community diversity. A higher number represents more diversity;

The biodiversity indices (OTUs, Chao1, PD, Shannon and Simpson) were calculated based on normalization of the number of the filtered sequences from each sludge sample to 40000 .

Fig. 3 showed the changes in diversity of key bacteria at start-up (inoculum), at the 43rd operation (A1), and at the end of operation (A2). Clear differences in the relative abundances were observed as EGSB reactor operation continued. At the bacterial phylum level, there was an apparent increase in relative sequences of Chloroflexi and Euryarchaeota (33.01\%, 0.63\% in A1 and 44.03\%, 7.14\% in A2), which was $19.40 \%$ and $0.54 \%$ in the inoculum. This increase was in accordance with the previous work of Chen et al. ${ }^{[15]}$. Meanwhile, the vast majority of the total sequence detected in inoculum such as Proteobacteria (23.30\%), Actinobacteria (8.68\%), Acidobacteria (4.86\%), Bacteroidetes (10.80\%), Gemmatimonadetes (1.24\%) and TM7 (1.32\%) decreased in A1 and A2.

At the genus level, except unclassified genus, Methanosarcina, Clostridium and WCHB1-05 were found to be the main members, whose sum occupied $16.13 \%$ and $18.41 \%$ of the total sequences in A1 and A2, respectively. In particular, there was a significant increase in Methanosarcina and WCHB1-05 (6.46\% and $5.87 \%$ in A2), which was only $0.05 \%$ and $1.03 \%$ in the inoculum. Meanwhile, the relative abundances of other genera such as Hyphomicrobium, Methanobacterium, Methylosinus, Caldilinea, Turicibacter, Rhodoplanes, Nannocystis, Proteiniclasticum, Gemmata, Novosphingobium, Crenothrix, and Nitrospira decreased in A1 andA2. EGSB reactor under the condition of poor nutrition is successfully start up, and the inoculation sludge was flocculent sludge from anoxic area instead of granular sludge. It indicated the EGSB reactor had a good adaptability, and the diversity of microbial community was also in line with the actual situation. 


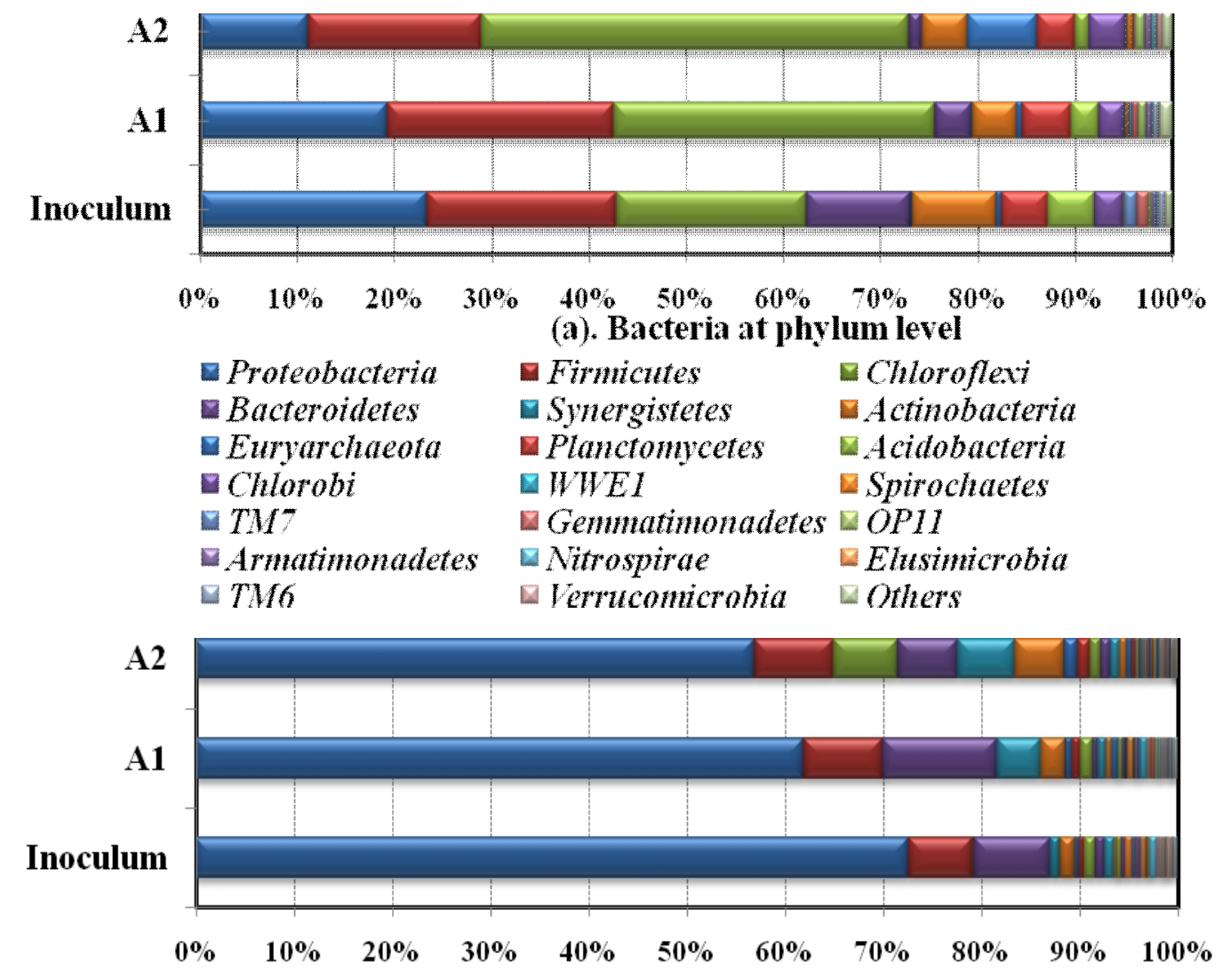

(b). Bacteria at genus level

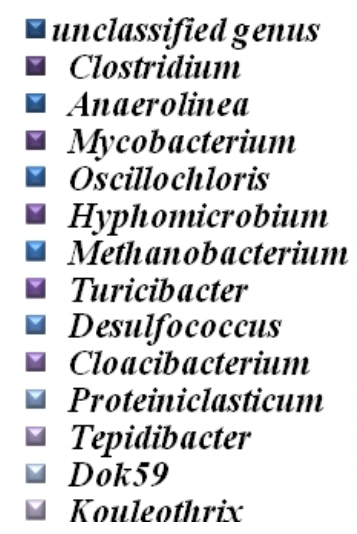

$\square$ others $(<1 \%)$
$\square$ WCHB1-05
$\square$ SHD-231
$\square$ Caldilinea
$\square$ DCE29
$\square$ Chlorobaculum
$\square$ Treponema
$\square$ Dechloromonas
$\square$ Bacillus
$\square$ Nannocystis
$\square$ Genmata
$\square$ Nitrospira
$\square$ Novosphingobium
$\square$ Crenothrix

$\square$ Methanosarcina
$\square$ Longilinea
$\square$ Methylosinus
$\square T 78$
$\square$ A17
$\square$ Planctomyces
$\square$ Desulfomonile
$\square$ Rhodoplanes
$\square$ Pirellula
$\square$ Epulopiscium
$\square$ E6
$\square$ Thermomonas
$\square$ Devosia
$\square$ Geothrix

Fig. 3. Changes in diversity of key microbiology community by next generation sequencing $(\mathrm{A} 1=$ samples were taken after 43rd days of an EGSB operation, and A2 = samples were taken at the end of an EGSB operation).

\section{Conclusions}

poor nutrition simulated wastewater with sodium acetate carbon source in an EGSB reactor successfully start up, and EGSB reactor inoculated the flocculent sludge from the anoxic zone in the municipal sewage treatment plant. The results demonstrated the COD removal efficiency can achieve more than $90 \%$, when the COD concentration of influent was improved $1000 \mathrm{mg} / \mathrm{L}$ in the continuous operation of 73 days. The NGS results showed there was an apparent increase in relative sequences of Chloroflexi and Euryarchaeota $(33.01 \%, 0.63 \%$ in A1 and $44.03 \%, 7.14 \%$ in A2), which was $19.40 \%$ and $0.54 \%$ in the inoculum at the bacterial phylum level. there was a significant increase in Methanosarcina and 
WCHB1-05 (6.46\% and 5.87\% in A2), which was only $0.05 \%$ and $1.03 \%$ in the inoculum at the genus level.

\section{Acknowledgements}

This work was financially supported by the Natural Science Foundation of China (No. 51468024), Doctor Start Funding Project and College Students Innovation Training Program of Jingdezhen Ceramic Institute.

\section{References}

[1] G. Lettinga,et al. Anaerobia treatment of sewage and low strength wastewater. Proc Anaerobic Digestion. Elsevier Biomedical Press, Amsterdam (1981)

[2] M. T. Kato. The anaerobic treatment of low strength soluble wastewaters. Wageningen Agricultural University.

[3] L. A. Nunez,et al. Anaerobic treatment of slaughterhouse wastewater in an expanded granular sludge bed (EGSB)reactor. Wat Sci Tech, ,40(8):99 106 (1999)

[4] G. R. Zoutberg and R. Frankin. Anaerobic treatment of chemical and brewery wastewater with a new type of anaerobic reactor: the BiobedÒEGSB reactor. Wat Sci Tech ,34(5 6):375 381(1996)

[5] R. Liao, K.Shen, and A. Li, et al.,. Highnitrate wastewater treatment in an expanded granular sludge bed reactor and microbial diversity using 454 pyrosequencing analysis. Bioresour. Technol. 134, 190-197(2013)

[6] American Public Health Association (APHA), American Water Works Association (AWWA), Water Environment Federation (AEF). Standard Methods for the Examination of Water and Wastewater, 21st ed., Washington, DC, USA. (2005)

[7] J.G. Caporaso, et al., Ultra-high-throughput microbial community analysis on the Illumina HiSeq and MiSeq platforms, ISME J. 6 (8) 1621-1624(2012).

[8]. X. Xie, N. Liu, and B. Yang, Chengzhi Yu, Qingyun Zhang, Xiulin Zheng, Leyi Xu, Ran Li, Jianshe Liu. Comparison of microbial community in hydrolysis acidification reactor depending on different structure dyes by Illumina MiSeq sequencing. International Biodeterioration \& Biodegradation 111 14e21 (2016).

[9] Y. Yin, and J. Wang. Changes in microbial community during biohydrogen production using gamma irradiated sludge as inoculums. Bioresource Technology 200 217-222 (2016)

[10] N. Fierer, et al., Cross-biome metagenomic analyses of soil microbial communi- ties and their functional attributes, Proc. Natl. Acad. Sci. U. S. A. 109 (52), 21390-21395 (2012)

[11] P. D. Schloss, D. Gevers, and S. L. Westcott. Reducing the effects of PCR amplification and sequencing artifacts on 16S rRNA-based studies. PloS one 6, e27310 (2011).

[12] K. R. Amato, C. J. Yeoman, and A. Kent, et al., Stumpf, R.M., Yildirim, S., Torralba, M., Gillis, M., Wilson, B.A., Nelson, K.E., White, B.A., Leigh, S.R.,. Habitat degradation impacts black howler monkey (Alouatta pigra) gastrointestinal microbiomes. ISME J. 7, 1344e1353(2013).

[13] I. Colussi, A. Cortesi, and L. Della Vedova, et al., Start-up procedures and analysis of heavy metals inhibition on methanogenic activity in EGSB reactor. Bioresource Technology, 100(24) : 6290-6294 (2009)

[14] J. Liu, J. Hu, and J. Zhong, et al., The effect of calcium on the treatment of fresh leachate in an expanded granular sludge bed bioreactor. Bioresource Technology, 102,(9): 5466-5472(2011)

[15] C.L. Chen, J.H. Wu, and W.T. Liu, Identification of important microbial populationsin the mesophilic and thermophilic phenol-degrading methanogenicconsortia, Water Res. 42:1963-1976. (2008) 